\title{
Regulation of aminopeptidase A expression in cervical carcinoma: role of tumor-stromal interaction and vascular endothelial growth factor
}

\author{
Takayasu Suganuma ${ }^{1}$, Kazuhiko Ino ${ }^{1}$, Kiyosumi Shibata ${ }^{1}$, Seiji Nomura ${ }^{1}$, Hiroaki \\ Kajiyama $^{1}$, Fumitaka Kikkawa ${ }^{1}$, Nobuo Tsuruoka ${ }^{2}$ and Shigehiko Mizutani ${ }^{1}$ \\ ${ }^{1}$ Department of Obstetrics and Gynecology, Nagoya University Graduate School of Medicine, Nagoya, Japan \\ and ${ }^{2}$ Institute for Health Care Science, Suntory Ltd, Osaka, Japan
}

\begin{abstract}
We previously demonstrated that aminopeptidase A (APA), a membrane-bound metallopeptidase degrading bioactive peptides such as angiotensin II (Ang II), is expressed in neoplastic lesions of the uterine cervix, and that its expression is upregulated as the lesion progresses from cervical intraepithelial neoplasms (CIN) toward invasive squamous cell carcinomas (SCC). The present study investigated the regulatory mechanisms involved in APA expression and its potential role in cervical carcinoma. Immunohistochemical staining in high-grade CIN and SCC tissues showed that APA was strongly expressed at the edge of lesions adjacent to cervical stromal cells. Fluorescence-activated cell sorting analysis demonstrated that cell surface APA expression was extremely low in three human SCC cell lines, SiHa, TCS and CaSki, under basal conditions. However, both contact and noncontact cocultures with human cervical fibroblasts resulted in the induction of APA expression in these SCC cells. APA expression was also induced in vivo when TCS cells were subcutaneously inoculated into nude mice. Furthermore, APA expression and enzymatic activity were enhanced by addition of the conditioned medium (CM) from fibroblast culture, but not by heat-treated CM. Among the various cytokines tested, vascular endothelial growth factor (VEGF) significantly increased APA activity, and induction of APA by the fibroblast CM was partly inhibited by anti-VEGF neutralizing antibody. Finally, APA cDNA-transfected APAoverexpressing TCS cells significantly reduced the Ang Il-induced cell invasion ability as compared with parental or control vector-transfected TCS cells, although there was no significant difference in cellular proliferation among them. These results suggested the importance of tumor-stromal interaction for the regulation of APA expression in the microenvironment of cervical carcinoma and the potential role for this peptidase in regulating tumor invasion through inactivation of Ang II activity.
\end{abstract}

Laboratory Investigation (2004) 84, 639-648, advance online publication, 29 March 2004; doi:10.1038/labinvest.3700072

Keywords: aminopeptidase A; angiotensin II; cervical carcinoma; fibroblast; invasion; VEGF

Cell surface peptidases play a key role in the control of growth, differentiation and signal transduction of many cellular systems by modulating the activity of bioactive peptides and regulating their access to receptors or adjacent cells., ${ }^{1,2}$ Thus, abnormal changes in expression pattern and catalytic function of peptidases result in altered peptide activation, which disrupts normal cellular homeostasis and may contribute to neoplastic transformation or tumor

Correspondence: Dr Kazuhiko Ino, MD, Department of Obstetrics and Gynecology, Nagoya University Graduate School of Medicine, 65 Tsurumai-cho, Showa-ku, Nagoya 466-8550, Japan.

E-mail: kazuino@med.nagoya-u.ac.jp

Received 2 October 2003; revised 20 December 2003; accepted 22 December 2003; published online 29 March 2004 progression. Recent studies have demonstrated that several membrane-bound peptidases, including neutral endopeptidase (NEP), ${ }^{3,4}$ dipeptidyl peptidase IV (DPPIV) ${ }^{5,6}$ and aminopeptidase $\mathrm{N}$ (APN), ${ }^{7,8}$ are involved in tumor cell growth, differentiation and invasion in various human malignancies through upregulation or downregulation of their expression dependent on the types of malignancies.

Aminopeptidase A (APA, angiotensinase, EC 3.4.11.7) is also a zinc-dependent membrane-bound peptidase, which specifically cleaves the aminoterminal glutamyl or aspartyl residue from peptide substrates such as angiotensin II (Ang II). ${ }^{9}$ APA has recently been cloned and proven to be identical to the mouse B-lymphocyte lineage marker $\mathrm{BP}-1^{10}$ or the human kidney differentiation antigen gp160. ${ }^{11}$ 
Physiologically, APA is expressed in vascular pericytes $^{12,13}$ and renal proximal tubules/glomeruli, $^{14}$ and involved in the regulation of blood pressure by converting Ang II to Ang III in the catabolic pathway of the renin-angiotensin system. ${ }^{15}$ We purified APA from the human placenta ${ }^{16}$ and demonstrated the role of this enzyme in regulating blood pressure during pregnancy. ${ }^{17}$ However, there have been few reports on APA expression and its functional significance in tumor cells. ${ }^{18-20}$

We have recently demonstrated that APA was expressed in neoplastic lesions of the uterine cervix, and its expression was upregulated as the lesion progresses from cervical intraepithelial neoplasms (CIN) toward invasive squamous cell carcinomas (SCC). ${ }^{21}$ However, the regulatory mechanisms for APA expression and its functional role in the progression of cervical carcinoma remain to be clarified. It has been suggested that tumor-stromal interaction is a critical factor in both tumor invasion and proliferation, and the stromal cells play a key role in tumor cell invasion by providing specific soluble factors and extracellular matrix (ECM) molecules. ${ }^{22,23}$ In fact, induction of matrix metalloproteinases (MMPs) and their activation, which are essential for ECM degradation, are demonstrated by contact with stromal fibroblasts or macrophages. ${ }^{24,25}$ Based on these findings, in the present study, we cocultured cervical cancer cells with cervical fibroblasts in vitro under contact or noncontact conditions, mimicking in vivo tumor-stromal interactions. Using these systems, we examined the APA expression levels and attempted to identify factors responsible for APA induction in cervical carcinoma. Furthermore, we transfected APA cDNA into cervical cancer cells to obtain APA-overexpressing cells, and attempted to clarify the role for APA in the invasiveness and proliferation of cervical cancer cells in the presence or absence of its specific peptide substrate Ang II.

\section{Materials and methods}

\section{Reagents and Antibodies}

Human Ang II was purchased from the Peptide Institute (Osaka, Japan). Human recombinant vascular endothelial growth factor (VEGF), transforming growth factor (TGF)- $\beta 1$, tumor necrosis factor (TNF)- $\alpha$, interferon (IFN)- $\gamma$, and interleukin (IL)- $1 \beta$ were all purchased from Sigma-Aldrich (St Louis, MO, USA).

Rabbit anti-APA polyclonal antibody was raised against a synthetic peptide composed of the 18amino-acid sequence from $\mathrm{Ser}^{257}$ to $\mathrm{Thr}^{274}$ (SNMPVAKEESVDDKWTRT) in human APA as previously described. ${ }^{20}$ Mouse monoclonal antibody S4 (URO-2) specific to human APA was obtained from Signet Laboratories (Dedham, MA,
USA). Anti-VEGF neutralizing antibody was purchased from Santa Cruz Biotechnology (Santa Cruz, CA, USA).

\section{Cell Lines and Culture}

Human cervical cancer (SCC) cell lines, SiHa, TCS and CaSki, were used. SiHa and CaSki were purchased from ATCC (Manassas, VA, USA). TCS was a generous gift from RIKEN (Saitama, Japan). Human choriocarcinoma cell line BeWo was also purchased from ATCC. All cell lines were maintained in DMEM or RPMI medium supplemented with $10 \%$ fetal calf serum (FCS) and penicillin/ streptomycin and incubated at $37^{\circ} \mathrm{C}$ in a humidified atmosphere of $5 \% \mathrm{CO}_{2}$.

Human fibroblasts were obtained from tissue specimens of the uterine cervix, which were surgically resected from patients with benign gynecologic diseases under informed consent. Primary cultures of fibroblasts were established as previously described ${ }^{22}$ with minor modifications. Briefly, cervical stromal tissues were finely minced and digested with collagenase and trypsin. Then the suspension was passed through a $200 \mu \mathrm{m}$ pore nylon mesh to remove undigested fragments. The collected cells were washed with phosphate-buffered saline (PBS) and cultured in DMEM with $10 \%$ FCS. Fibroblasts were confirmed by immunostaining with monoclonal antibodies to cytokeratin 14 and vimentin (Dako, Carpintaria, CA, USA) as previously described. ${ }^{22}$ Fibroblasts were cultured up to 10 passages and were used at passages three to five. The conditioned medium (CM) was collected from subconfluent cultures of the fibroblasts after incubation in six-well culture plates $\left(1 \times 10^{5}\right.$ cells/well $)$ in serum-free medium for $24 \mathrm{~h}$.

\section{Co-culture Experiments}

In contact co-culture experiments, cervical cancer cells $\left(1 \times 10^{4}\right.$ cells/well $)$ alone or fibroblasts $\left(2 \times 10^{4}\right.$ cells/well) alone or in combination were seeded in four-well chamber glass slides (LabTek, Nunc Inc., Naperville, IL, USA), and cultured for $24 \mathrm{~h}$ in serum-free medium. The cells were washed with PBS and fixed with $4 \%$ paraformaldehyde, and immunocytochemical staining was performed as described below. Noncontact co-culture experiments were performed using 24-well transwell chambers with a $0.4 \mu \mathrm{m}$ pore membrane (Corning, NY, USA). Different numbers of fibroblasts $\left(1 \times 10^{4}\right.$ or $1 \times 10^{5}$ cells) were seeded in the upper compartments, while cervical cancer cells $\left(5 \times 10^{4}\right.$ cells $)$ were seeded in the lower compartments. After incubation in serum-free medium for $24 \mathrm{~h}$, APA activity in cervical cancer cells was measured. In negative controls, medium alone (no fibroblast) was added to the upper compartments. 


\section{Immunohistochemistry and Immunocytochemistry}

Cervical neoplastic tissues including high-grade CIN (CIN III, $n=5$ ) and invasive SCC $(n=10)$ were obtained from patients who underwent hysterectomy at Nagoya University Hospital. The use of tissue samples was approved by the Ethics Committee of Nagoya University Hospital and the individual patients, respectively. Formalin-fixed, paraffinembedded tissue sections were cut at a thickness of $4 \mu \mathrm{m}$. Immunohistochemical staining was performed using the avidin-biotin immunoperoxidase technique as previously described. ${ }^{21}$ The primary antibody (anti-APA) at a dilution of 1:200 was used and incubated at room temperature for $2 \mathrm{~h}$. The sections were rinsed with $\mathrm{PBS}$ and incubated for $30 \mathrm{~min}$ with biotinylated second antibody. After washes with PBS, the sections were incubated for $30 \mathrm{~min}$ with horseradish peroxidase-conjugated streptavidin, and finally treated with 3-amino-9ethylcarbazole (AEC) in PBS containing $0.01 \% \mathrm{H}_{2} \mathrm{O}_{2}$ for $10 \mathrm{~min}$. The slides were counterstained with Meyer's hematoxylin. As a negative control, the primary antibody was replaced with normal rabbit IgG at an appropriate dilution. Immunocytochemistry for APA expression in co-cultured cells was also performed using the same avidin-biotin immunoperoxidase method.

\section{Tumor Cell Inoculation into Nude Mice}

TCS or SiHa cells $\left(5 \times 10^{6}\right.$ cells $/ 0.5 \mathrm{ml}$ of medium $)$ were inoculated subcutaneously in the right flank of 5-week-old female nude mice (BALB/c) (Chubu Kagaku, Nagoya, Japan). The animals $(n=3)$ were kept in a temperature-controlled room with food and water. All mice developed a single subcutaneous palpable tumor 1 week after cell inoculation. At 4 weeks after inoculation, tumors were resected from mice, fixed in formalin, and embedded in paraffin for subsequent immunohistochemistry.

\section{Flow Cytometric Analysis}

Fluorescence-activated cell sorting (FACS) was performed to quantify cell-surface APA expression. Cells $\left(1 \times 10^{6} / \mathrm{ml}\right)$ were stained with mouse antiAPA monoclonal antibody S4 (diluted 1:40) or the isotype-matched control mouse IgG (Coulter, Hialeah, FL, USA) for $1 \mathrm{~h}$ at $4^{\circ} \mathrm{C}$. The cells were washed three times and incubated with phycoerythrin (PE)-conjugated goat anti-mouse IgG (Coulter, 1:80 dilution) for $30 \mathrm{~min}$ at $4^{\circ} \mathrm{C}$. After washing, FACS data were acquired on a FACS Calibur (Becton Dickinson, San Jose, CA, USA) and analyzed using CELLQuest software (Becton Dickinson). In all, 10000 cells were analyzed in one parameter mode.

\section{Assay for APA Enzymatic Activity}

APA enzymatic activity was measured spectrophotometrically as previously reported..$^{20}$ Cells $\left(1 \times 10^{5} /\right.$ well) were suspended in 96-well microtiter plates in $200 \mu \mathrm{l}$ of substrate solution consisting of $1.5 \mathrm{mM} \quad \alpha$-L-glutamic acid- $p$-nitroanilide (Peptide Institute) in $0.1 \mathrm{M}$ Tris $\mathrm{HCl}(\mathrm{pH} 7.0)$ containing $2.5 \mathrm{mM} \mathrm{CaCl}{ }_{2}$. Following incubation at $37^{\circ} \mathrm{C}$ for 30 min with continuous agitation, the reaction was terminated by the addition of ice-cold PBS. APA activity was measured at $405 \mathrm{~nm}$ by a microplate reader. Cell- and substrate-free blanks were run in parallel.

\section{Western Blotting}

Cells were lysed in a lysis buffer consisting of $20 \mathrm{mM}$ Tris $\mathrm{HCl} \mathrm{pH}$ 7.5, $2 \mathrm{mM}$ EDTA, $150 \mathrm{mM} \mathrm{NaCl}$, $1 \%$ Triton X-100 and the protease inhibitor mixture. After centrifugation at $15000 \mathrm{~g}$ for $30 \mathrm{~min}$, the supernatant was obtained. Protein extract $(20 \mu \mathrm{g})$ was separated by SDS/7.5\% polyacrylamide gel electrophoresis (PAGE), transferred onto nitrocellulose membranes, and immunoblotted with anti-APA polyclonal antibody. Immunoreactive proteins were stained using a chemiluminescence detection system (ECL, Amersham Pharmacia Biotech).

\section{Plasmid Construction and Stable Transfection}

Full-length cDNA for human APA was prepared as described previously. ${ }^{26}$ TCS cells were transfected with eukaryotic expression vector pcDNA3.1(-) (TCS-pcDNA cells) (Invitrogen, Carlsbad, CA, USA) or pcDNA3.1(-) with APA cDNA inserted (TCS-APA cells) using lipofectamine according to the manufacturer's instructions (Life Technologies, San Diego, CA, USA). Stable transfectants were selected by growth in media supplemented with $400 \mu \mathrm{g} / \mathrm{ml}$ of G418 (Sigma-Aldrich) and clones resistant to G418 were obtained as APA-overexpressing cells. A total of 100 stable clones were obtained. The APA expression level on each transfectant was evaluated by Western blot analysis and enzyme activity assay, and several clones highly expressing APA were selected and used.

\section{In vitro Cell Proliferation Assay}

Cells (1500 cells/well) were plated in triplicate in 96-well culture plates with or without various concentrations of Ang II and cultured for 24-48 h. Cell viability was assayed using a modified MTT protocol by CellTiter 96 Aqueous One Solution Cell Proliferation Assay kit (Promega, Madison, WI, USA) according to the manufacturer's instructions. Absorbance was measured at $490 \mathrm{~nm}$ by a microplate reader. 


\section{In vitro Cell Invasion Assay}

Cell invasion was evaluated using 24-well Matrigel invasion chambers (Becton Dickinson). Cells were suspended in the upper chamber at a density of $4 \times 10^{4}$ cells in $200 \mu \mathrm{l}$ of media with various concentrations of Ang II. The lower chamber contained $750 \mu \mathrm{l}$ of D-MEM supplemented with 10\% FCS as a chemoattractant. After incubation for $16 \mathrm{~h}$, the remaining tumor cells on the upper surface of the filters were removed by wiping with cotton swabs, and invading cells on the lower surface were stained using May-Grünwald Giemsa. The number of cells on the lower surface of the filters was counted under a microscope. Data were obtained from three individual experiments in triplicate.

\section{Statistical Analysis}

Each experiment was performed in triplicate, and the results are presented as the mean \pm s.d. of three independent experiments. Analysis of variance (ANOVA) with Bonferroni corrections was applied to compare the difference between experimental groups, and $p<0.05$ was considered significant.

\section{Results}

\section{Immunohistochemical Expression of APA in CIN and SCC Tissues}

We first confirmed the immunohistochemical expression of APA in tissue samples of cervical neoplasia. As demonstrated in our previous paper, ${ }^{21}$ APA was expressed in all high-grade CIN $(n=5)$ and invasive SCC $(n=10)$ tissues examined. Interestingly, in most cases, we found that APA was strongly expressed on tumor cells located at the edges of lesions surrounded by adjacent cervical stromal cells (Figure 1a and b).
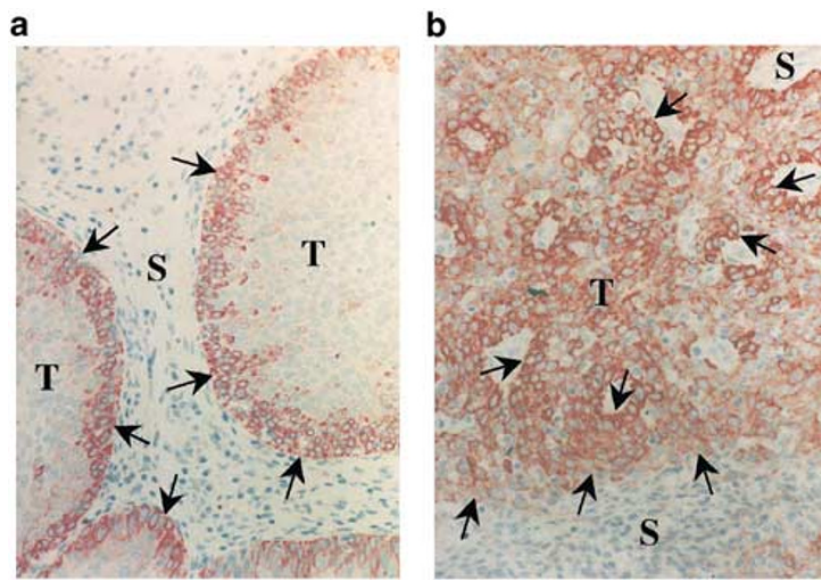

Figure 1 Immunohistochemical expression of APA in tissue sections of high-grade CIN (a) and invasive SCC (b). APA was strongly expressed at the edges of lesions (arrows) surrounded by adjacent cervical stromal cells. T, tumor; S, stroma. Original magnification, $\times 100$ in (a), $\times 200$ in (b).

\section{Expression of APA in Cervical Cancer Cell Lines}

Next, we examined cell-surface expression of APA in human cervical cancer (SCC) cell lines by FACS analysis. As shown in Figure 2, all three SCC cell lines, TCS, SiHa and CaSki, showed extremely low or almost no APA expression on the cell surface under the normal culture conditions, while BeWo choriocarcinoma cells, used as a positive control, showed strong APA expression as previously reported. ${ }^{20}$

\section{Induction of APA Expression by Contact with Stromal Fibroblasts}

To clarify possible mechanisms involved in the regulation of APA expression in cervical carcinoma, cervical cancer cells were co-cultured with fibroblasts derived from the uterine cervical stroma as a model of the in vivo microenvironment at the tumor-stromal interface. As shown in Figure 3a-e, contact co-cultures of TCS or SiHa cells with cervical fibroblasts resulted in a marked induction of APA expression in these SCC cells as demonstrated by immunocytochemistry, while either tumor cells or fibroblasts alone showed faint or no expression of APA. In addition, APA expression was also clearly detected in vivo in tumor cells surrounded by mouse stromal fibroblasts after TCS cells were subcutaneously inoculated into nude mice and developed for 4 weeks to form a tumor mass (Figure 3f). Similar APA induction was also observed when SiHa cells were inoculated into nude mice (data not shown). These results suggested that APA expression was upregulated by contact with adjacent stromal fibroblasts.

\section{APA Upregulation by Fibroblast CM}

To determine whether APA expression could be induced by a soluble factor(s) or direct cell-cell contact, we examined APA expression by cervical cancer cells in non-contact co-cultures with fibroblasts, or in cultures with CM from fibroblast culture. APA enzymatic activity in TCS or SiHa cells was significantly enhanced by transwell co-cultures with fibroblasts (Figure 4a). Furthermore, the fibroblast CM significantly enhanced APA activity in a concentration-dependent manner (Figure 4b). These results suggest that soluble factor(s) produced by cervical fibroblasts were mainly involved in the induction of APA expression. Induction of APA protein expression in TCS and SiHa cells by treatment with fibroblast CM was also demonstrated by immunocytochemistry (Figure 4c) and Western blotting (Figure 4d). 
Control IgG

APA
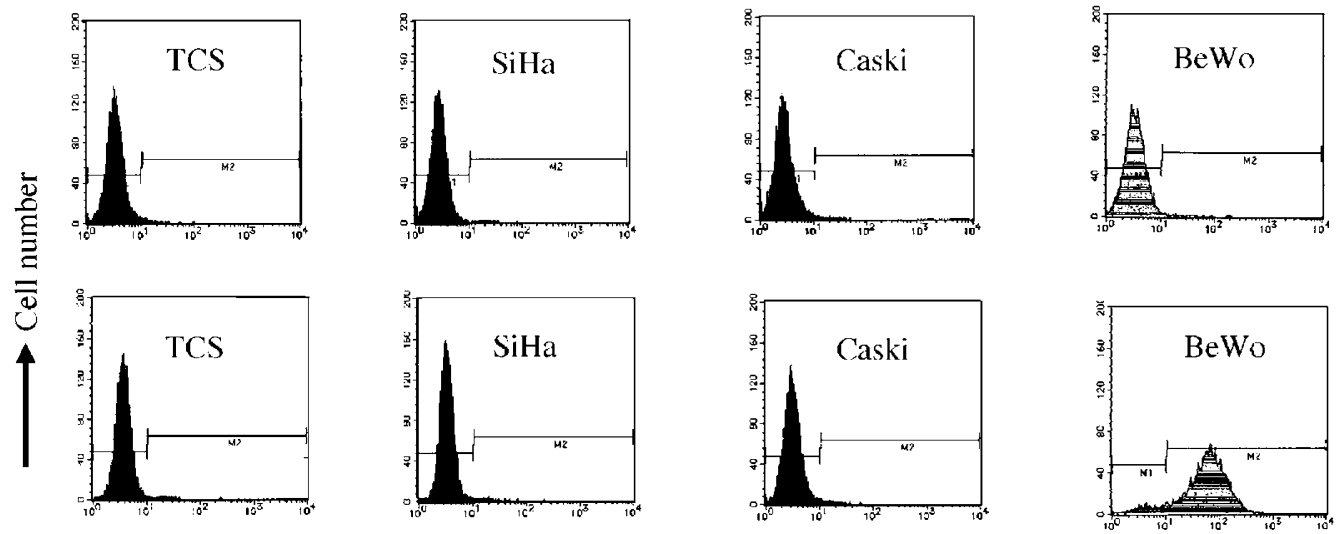

Log fluorescence intensity

Figure 2 Cell-surface expression of APA in cervical cancer cell lines. Cells were stained with APA-specific mouse monoclonal antibody (lower panels) or isotype-matched control mouse IgG (upper panels), followed by staining with PE-conjugated antibody. FACS analysis was performed on TCS, SiHa, CaSki, and also a choriocarcinoma cell line BeWo used as a positive control. Note: In all SCC cell lines, there was almost no APA expression on the cell surface under basal culture conditions.

a

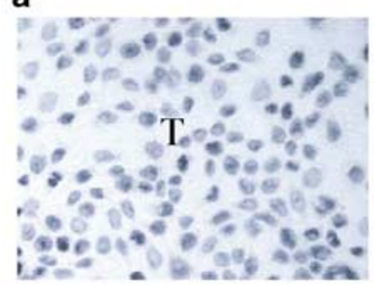

d

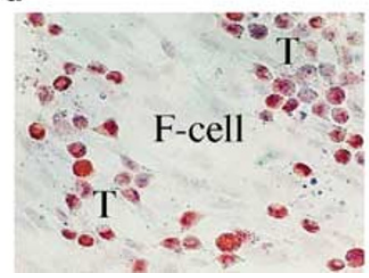

b

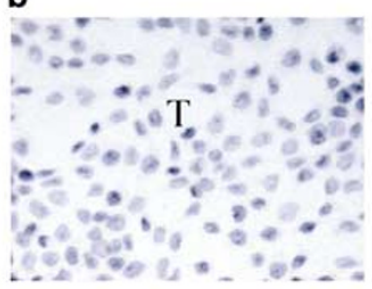

e

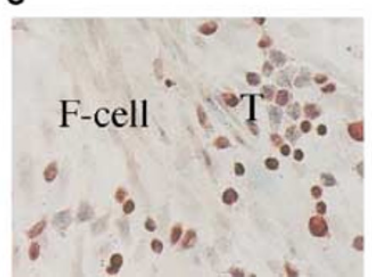

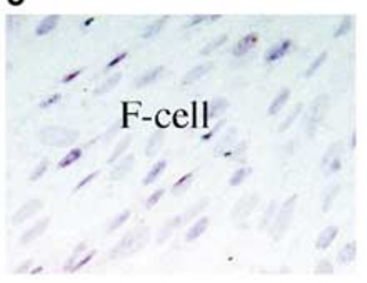

f

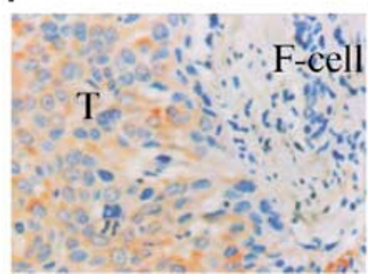

Figure 3 Immunocytochemical expression of APA in TCS cells alone (a), SiHa cells alone (b), cervical fibroblasts alone (c), TCS cells cocultured with cervical fibroblasts for $24 \mathrm{~h}$ (d), and SiHa cells co-cultured with cervical fibroblasts for $24 \mathrm{~h}$ (e). (f) Immunohistochemical expression of APA in TCS cells 4 weeks after subcutaneously inoculated into nude mice. T, tumor cells; F-cell, fibroblasts. Original magnification, $\times 200$ in (a)-(f).

\section{VEGF Increased APA Expression in Cervical Cancer Cells}

To identify the possible factor(s) responsible for APA induction in cervical cancer cells, we performed heat treatment of the $\mathrm{CM}$ (boiling at $95^{\circ} \mathrm{C}$ for $5 \mathrm{~min}$ ) before use. Most cytokines/growth factors could be inactivated by this heat treatment, while low-molecular-weight bioactive peptides $(<3.0 \mathrm{kDa})$ remained active after treatment. ${ }^{27}$ As shown in Figure 5a, enhancement of APA activity by fibroblast CM was significantly (but not completely) inhibited by heat treatment, suggesting that factors mainly involved in APA induction may be cytokine(s) rather than low-molecular-weight peptides. Among the various cytokines tested, VEGF significantly increased APA activity in TCS cells, but others, including TGF- $\beta 1$, TNF- $\alpha$, IFN- $\gamma$ and IL- $1 \beta$, did not affect APA activity (Figure 5b). Furthermore, induction of APA by fibroblast CM was partly inhibited by anti-VEGF neutralizing antibody (Figure 5c). These data suggested that APA induction in cervical cancer cells was due, at least in part, to VEGF secreted at the tumor-stromal interface.

\section{Overexpression of APA Reduced Ang II-mediated Cell Invasion}

To investigate the role of APA in cervical carcinoma, we stably introduced human APA cDNA into TCS cells. APA-transfected TCS cells (TCS-APA) showed 
644
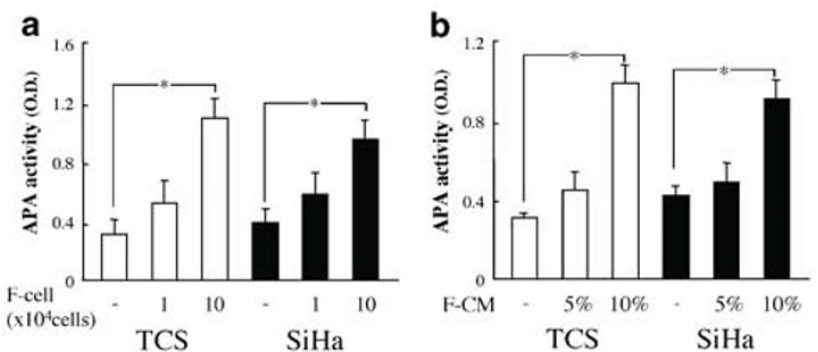

C

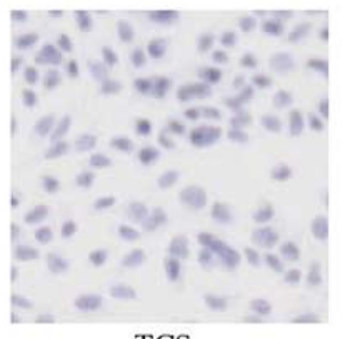

TCS

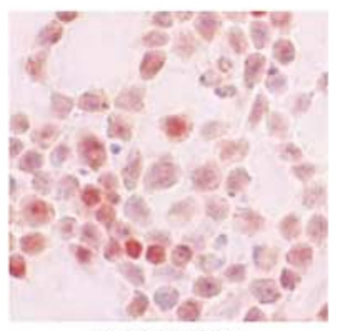

$\mathrm{TCS} / \mathrm{F}-\mathrm{CM}$

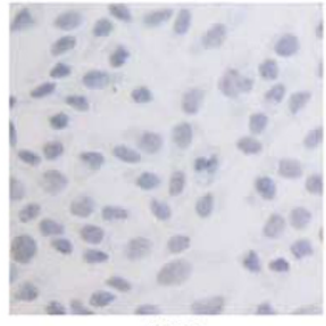

$\mathrm{SiHa}$

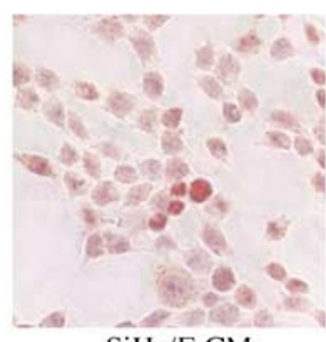

$\mathrm{SiHa} / \mathrm{F}-\mathrm{CM}$

d

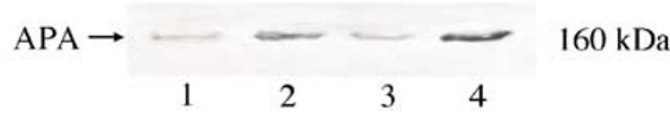

Figure 4 APA enzymatic activity in TCS and SiHa cells after noncontact co-cultures with fibroblasts (F-cell, $1 \times 10^{4}$ or $1 \times 10^{5}$ cells) for $24 \mathrm{~h}$ using the transwell chambers (a), or after incubation in serum-free media with or without fibroblast conditioned media (F-CM, $5 \%$ or $10 \%$ ) for $24 \mathrm{~h}$ (b). Data are expressed as the mean \pm s.d. of three independent experiments. ${ }^{*} P<0.05$. (c) Immunocytochemical expression of APA after incubation for $24 \mathrm{~h}$ in serum-free media with or without $10 \%$ F-CM. Magnification, $\times 200$. (d) Western blot analysis for APA expression in TCS (lane 1 and 2) and SiHa cells (lane 3 and 4). Cells were incubated for $24 \mathrm{~h}$ in serum-free media alone (lane 1 and 3 ) or in serum-free media with $10 \%$ F-CM (lanes 2 and 4). Note: APA was detected as a single band with a molecular mass of $160 \mathrm{kDa}$.

a high level of APA protein expression and remarkably increased APA activity as confirmed by Western blotting (Figure 6a) and enzyme assay (Figure 6b). Both parental (TCS) and control vectortransfected TCS cells (TCS-pcDNA) showed very low APA expression and enzymatic activity. There was no significant difference in cellular proliferation in the absence or presence of Ang II between TCS-APA and parental TCS or TCS-pcDNA (Figure 6c). In contrast, invasive potential in TCS-APA was significantly reduced as compared to those in TCS or TCS-pcDNA when the cells were incubated in the presence of Ang II, although there was no significant

a
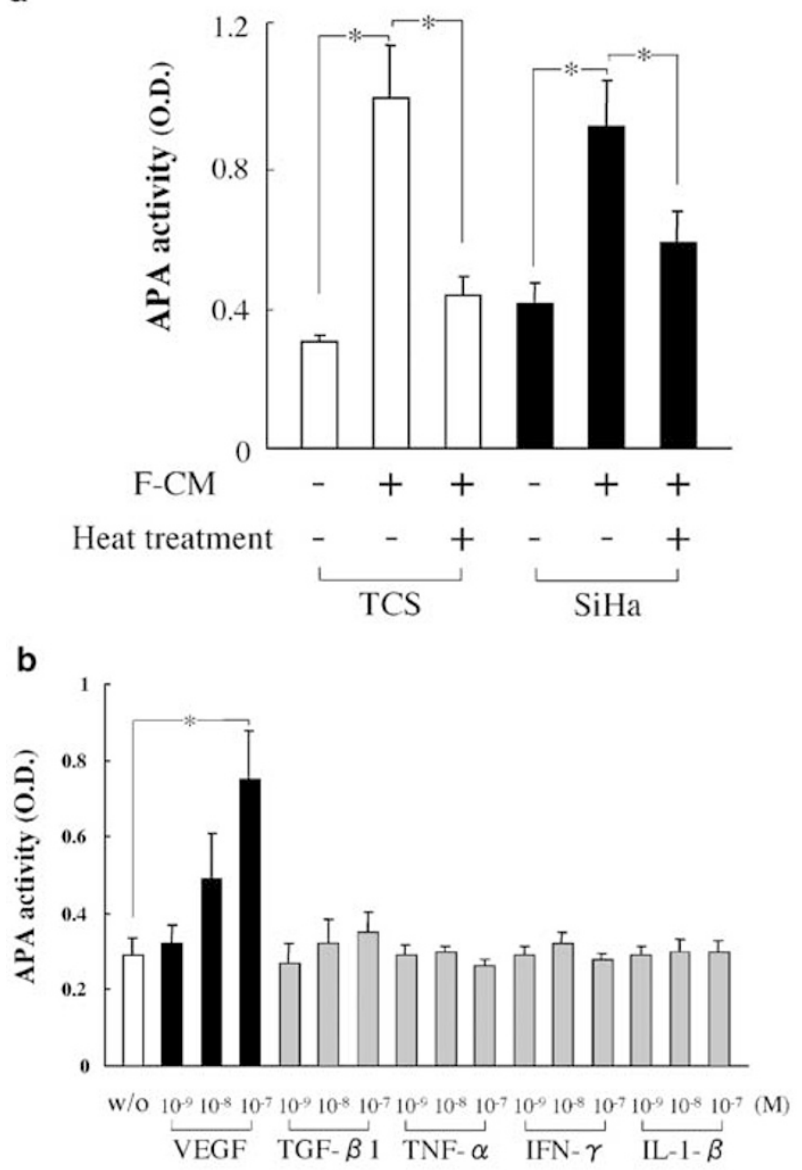

C

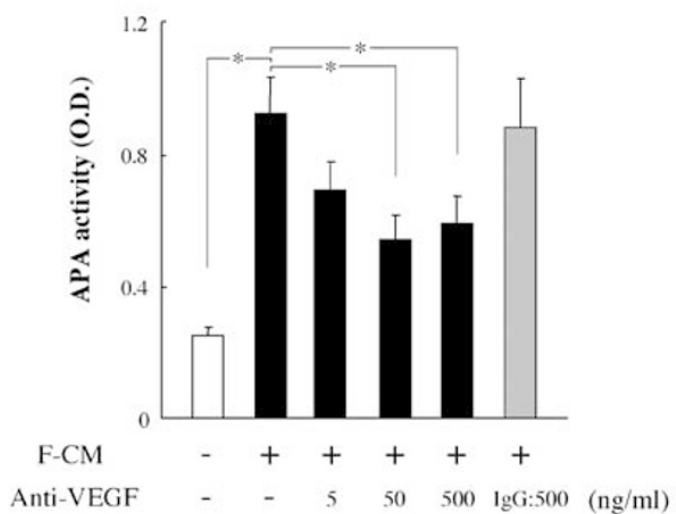

Figure 5 (a) APA enzymatic activity in TCS and SiHa cells after incubation for $24 \mathrm{~h}$ in serum-free media with untreated or heattreated fibroblast conditioned media (F-CM). (b) APA enzymatic activity in TCS cells after incubation for $24 \mathrm{~h}$ in serum-free media with the indicated concentrations of various cytokines. (c) APA enzymatic activity in TCS cells after treatment with $10 \%$ F-CM together with anti-VEGF neutralizing antibody $(5,50$ or $500 \mathrm{ng} /$ $\mathrm{ml})$ or control mouse IgG $(500 \mathrm{ng} / \mathrm{ml})$. All data are expressed as the mean \pm s.d. of three independent experiments. ${ }^{*} P<0.05$.

difference among the three groups when incubated in the absence of Ang II (Figure 6d). In addition, pretreatment of TCS cells with fibroblast CM significantly reduced the Ang II-induced cell invasion (Figure 7). These results suggest that APA may 
a

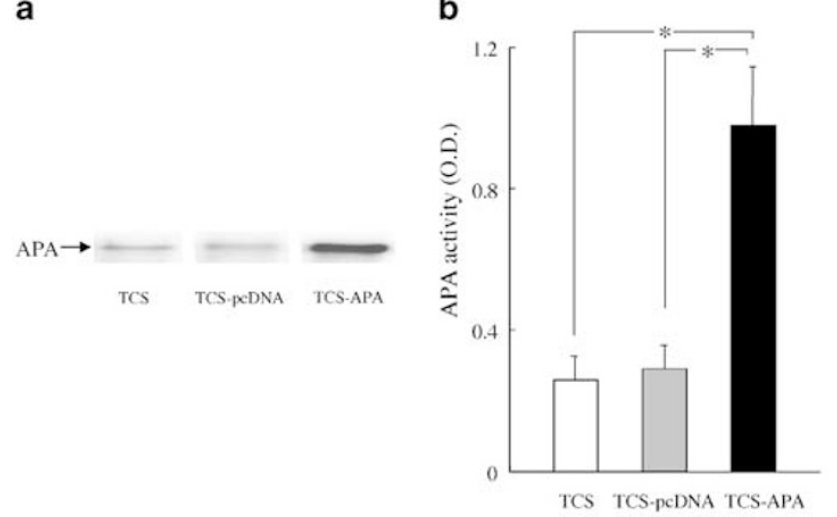

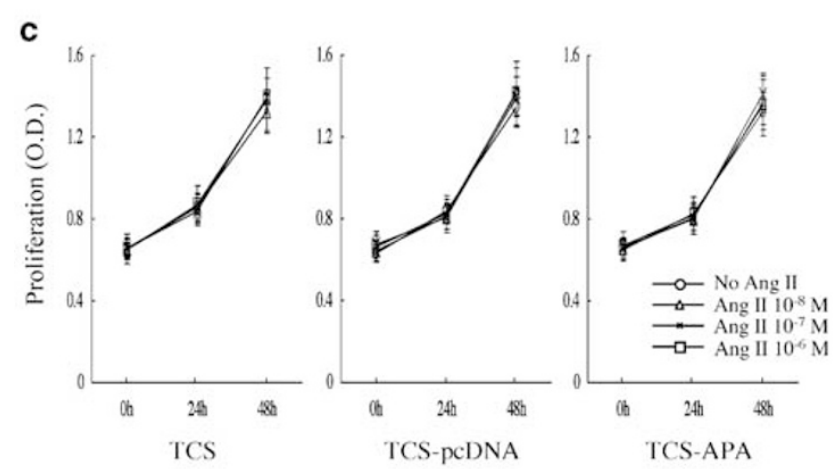

d

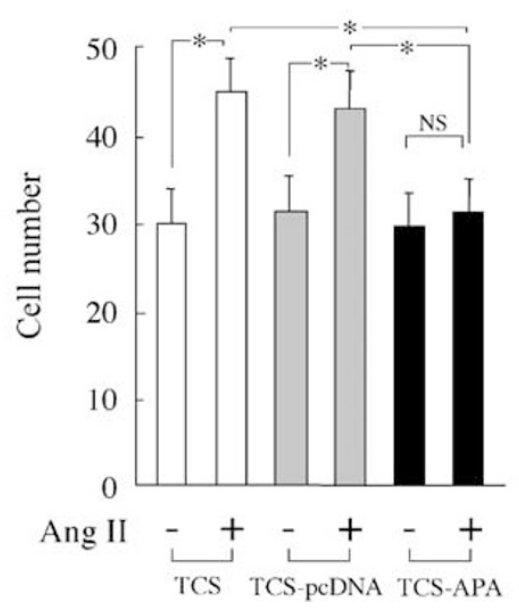

Figure 6 Overexpression of APA in APA cDNA-transfected TCS cells confirmed by Western blot analysis (a) and enzyme assay (b). TCS, parental TCS cells; TCS-pcDNA, TCS cells transfected with control vectors; TCS-APA, TCS cells transfected with APA expression vectors. (c) Cell proliferation was examined by MTT assay after incubation for 24 or $48 \mathrm{~h}$ with the indicated concentrations of Ang II. (d) Cell invasive ability after incubation for $16 \mathrm{~h}$ with or without Ang II at $10^{-7} \mathrm{M}$. All data are expressed as the mean \pm s.d. of three independent experiments. ${ }^{*} P<0.05$.

have a suppressive effect on Ang II-induced invasion of cervical cancer cells.

\section{Discussion}

We previously reported that APA was highly expressed in human cervical neoplastic lesions. ${ }^{21}$

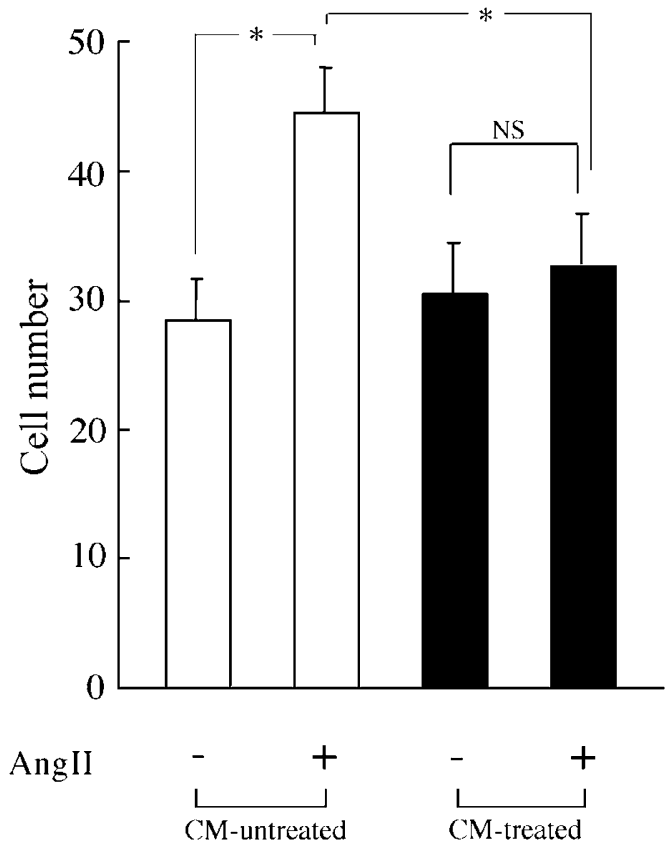

Figure 7 Effect of pretreatment with fibroblast CM on the invasive potential of TCS cells. TCS cells were pretreated (or untreated) with $10 \%$ fibroblast $\mathrm{CM}$ for $24 \mathrm{~h}$, and further incubated in invasion chambers for $16 \mathrm{~h}$ in the presence or absence of Ang II at $10^{-7} \mathrm{M}$. All data are expressed as the mean \pm s.d. of three independent experiments. ${ }^{*} P<0.05$.

Further detailed immunohistochemical analysis demonstrated that APA was strongly expressed at the edge of tumor lesions surrounded by adjacent cervical stromal cells. Based on these findings, the present study was focused on the role of interaction between tumor cells and stromal cells to clarify the regulatory mechanisms for APA expression in cervical carcinoma.

The present data demonstrated that co-cultures of cervical cancer cells with cervical fibroblasts markedly induced APA expression. Furthermore, it is of interest that APA expression was also induced in vivo when cervical cancer cells were inoculated into nude mice to form tumors surrounded by mouse fibroblasts. These results indicated that APA could be induced by interaction with stromal fibroblasts in the tumor microenvironment. Recent studies demonstrated that stromal cells surrounding tumor cells play a key role in tumor cell invasion and progression by providing specific soluble factors such as cytokines or ECM molecules. ${ }^{22,23}$ These tumor-stromal interactions are essential for MMP activation and ECM degradation for cancer invasion and metastasis. ${ }^{24,25,28}$ We reported that soluble factors derived from adjacent normal tissues enhanced MMP expression in ovarian cancer cells. ${ }^{29,30}$ Similarly, Bogenrieder et al ${ }^{31}$ reported that APA was expressed in stromal cells surrounding prostate cancer cells, but not in benign prostatic stroma. Saiki et $a l^{7}$ showed that another aminopeptidase APN was expressed at the invasive edge of 
renal cancer cells. Riemann et $a l^{32}$ also demonstrated that cell-cell contact with stromal cells was required for APN expression on tumor-infiltrating lymphocytes. These findings could support evidence that tumor-stromal interaction is essential not only for MMP activation, but also for the expression of aminopeptidases such as APA or APN during the tumor invasion and progression.

The current study demonstrated that a soluble factor(s), but not direct cell-cell contact, was required for APA induction in cervical cancer cells. Furthermore, our results from heat treatment experiments suggest that the factors involved in APA induction may be certain cytokine(s)/growth factor(s) rather than low-molecular-weight peptides. We checked the existence of cytokines in the fibroblast CM, and various cytokines, including IL$1 \beta$ IFN- $\gamma$, TGF- $\beta 1$, TNF- $\alpha$ and VEGF, were detected by enzyme-linked immunosorbent assay (ELISA) (data not shown). Previous studies reported that IFNs and IL-7 as well as the CM from bone marrow stromal cells upregulated APA expression in mouse B cells. ${ }^{33,34}$ In contrast, APA expression in renal carcinoma cells was upregulated by IFNs, while it was downregulated by TGF- $\beta 1$ and TNF- $\alpha .{ }^{35}$ APA expression in the brain tumor vasculature was also downregulated by TGF- $\beta .^{36}$ In the present study, among the cytokines tested, only VEGF significantly increased APA activity. Furthermore, APA induction was significantly (but not completely) inhibited by anti-VEGF neutralizing antibody. These data suggest that APA induction in cervical cancer cells is due mainly to VEGF secreted by the stromal cells. Similar VEGF-inducible aminopeptidase was found in mouse endothelial cells during angiogenesis. ${ }^{37}$ In contrast, we reported that APA was expressed in choriocarcinoma cells and its expression was upregulated by the peptide substrate Ang II. ${ }^{26}$ However, neither Ang II nor its receptor antagonist candesartan could affect APA expression levels in cervical cancer cells (data not shown). Thus, in addition to VEGF, some low-molecular-weight peptides (but not Ang II) might be involved, at least in part, in the upregulation of APA expression.

The functional significance of APA in cervical carcinoma remains to be determined. Enzymatically, APA specifically degrades and inactivates Ang II to generate Ang III in the catabolic pathway of the local renin-angiotensin system. Recent studies have shown that Ang II acts not only as a vasoconstrictor but also as a growth factor. ${ }^{38}$ Furthermore, Ang II stimulates cell migration. ${ }^{39}$ Indeed, we found that Ang II enhanced the growth of choriocarcinoma cells $^{26}$ and also stimulated the invasiveness of choriocarcinoma or cervical cancer cells (unpublished data). Thus, to clarify the role of APA in cervical cancer cells, we constructed APA-overexpressing cells and examined its behavior under conditions with or without Ang II. We found that invasive potential was significantly reduced in APA-overexpressing TCS cells when incubated in the presence of Ang II. Furthermore, pretreatment of TCS cells with the fibroblast CM (possibly with higher APA expression) significantly suppressed Ang II-induced cell invasion. These results suggest that APA may play a functional role as a negative regulator on Ang II-mediated cervical cancer invasion possibly via inactivation of locally produced Ang II. Thus, upregulation of APA by stromal cellderived factors (VEGF and others) might be one of the host defensive mechanisms against cancer progression in the activated local renin-angiotensin system. Similar to APA, our recent studies have shown that DPPIV, an aminopeptidase degrading certain chemokines, decreased the invasive potential of ovarian cancer cells, ${ }^{6}$ while another aminopeptidase APN had a stimulatory effect on invasion and ECM degradation in renal cancer or melanoma. ${ }^{7,40}$ Taken together, it appeared that aminopeptidases are involved in tumor cell invasion as either a stimulator or inhibitor, which might be dependent on their specific peptide substrates.

The present study showed that VEGF played a major role in upregulating APA expression in cervical cancer cells. Many studies demonstrated that VEGF is a key factor in stromal invasion as well as angiogenesis of cervical carcinoma in vivo. ${ }^{41-45}$ Immunohistochemically VEGF was localized to cervical tumor cells, and also moderately or weakly expressed in adjacent stromal cells. ${ }^{41,42,45}$ In the preliminary experiments, we found that VEGF levels in CM from three cervical cancer cell lines used in the present study were much lower than those in the cervical fibroblast CM (data not shown). Furthermore, APA expression was not induced in a culture of cervical cancer cells alone (Figures 2 and 3 ). Therefore, we speculated that APA expression in cervical cancer cells could be induced mainly by VEGF secreted from stromal fibroblasts, but not by VEGF from the tumor cell origin alone. Further studies are needed to clarify the detailed relationship between APA expression and VEGF at the tumor-stromal interface in vivo.

In summary, we demonstrated that APA expression in cervical cancer cells was upregulated by interaction with cervical stromal fibroblasts, and VEGF was mainly involved in this regulatory mechanism. Furthermore, overexpression of APA reduced Ang II-induced invasive ability. These findings indicate the importance of tumor-stromal interaction in regulating APA expression in the microenvironment of cervical carcinoma, and suggest the potential role for this peptidase in regulating tumor cell invasion through inactivation of Ang II activity.

\section{Acknowledgement}

This work was supported in part by Grant-in-Aid No. 15591742 (to KI) from the Japanese Ministry of Education, Culture, Sports, Science and Technology. 


\section{References}

1 Kenny AJ, O’Hare MJ, Gusterson BA. Cell-surface peptidases as modulators of growth and differentiation. Lancet 1989;2:785-787.

2 Shipp MA, Look AT. Hematopoietic differentiation antigens that are membrane-associated enzymes: cutting is the key!. Blood 1993;82:1052-1070.

3 Papandreou CN, Usmani B, Geng Y, et al. Neutral endopeptidase 24.11 loss in metastatic human prostate cancer contributes to androgen-independent progression. Nat Med 1998;4:50-57.

4 Suzuki T, Kikkawa F, Ino K, et al. Imbalance between neutral endopeptidase 24.11 and endothelin-1 expression in human endometrial carcinoma. Oncology 2001;60:258-267.

5 Wesley UV, Albino AP, Tiwari S, et al. A role for dipeptidyl peptidase IV in suppressing the malignant phenotype of melanocytic cells. J Exp Med 1999;190:311-322.

6 Kajiyama H, Kikkawa F, Khin EE, et al. Dipeptidyl peptidase IV overexpression induces up-regulation of E-cadherin and tissue inhibitors of matrix metalloproteinases, resulting in decreased invasive potential in ovarian carcinoma cells. Cancer Res 2003;63: 2278-2283.

7 Saiki I, Fujii H, Yoneda J, et al. Role of aminopeptidase $\mathrm{N}$ (CD13) in tumor-cell invasion and extracellu1ar matrix degradation. Int J Cancer 1993;54:137-143.

8 Ino K, Goto S, Okamoto $\mathrm{T}$, et al. Expression of aminopeptidase $\mathrm{N}$ on human choriocarcinoma cells and cell growth suppression by the inhibition of aminopeptidase N activity. Jpn J Cancer Res 1994; 85:927-933.

9 Glenner GG, McMillan PJ, Folk JE. A mammalian peptidase specific for the hydrolysis of N-terminal $\alpha$-L-glutamyl and aspartyl residues. Nature 1962; 194:867.

$10 \mathrm{Wu}$ Q, Lahti JM, Air GM, Burrows PD, Cooper MD. Molecular cloning of the murine BP-1/6C3 antigen: a member of the zinc-dependent metallopeptidase family. Proc Natl Acad Sci USA 1990;87:993-997.

11 Nanus DM, Engelstein D, Gastl GA, et al. Molecular cloning of the human kidney differentiation antigen gp 160: human aminopeptidase A. Proc Natl Acad Sci USA 1993;90:7069-7073.

12 Healy DP, Wilk S. Localization of immunoreactive glutamyl aminopeptidase in rat brain. II. Distribution and correlation with angiotensin II. Brain Res 1993;606:295-303.

13 Schlingemann RO, Oosterwijk E, Wesseling $\mathrm{P}$, et al. Aminopeptidase $\mathrm{A}$ is a constituent of activated pericytes in angiogenesis. J Pathol 1996;179:436-442.

14 Song L, Ye M, Troyanovskaya M, et al. Rat kidney glutamyl aminopeptidase (aminopeptidase A): molecular identity and cellular localization. Am J Physiol 1994;267:F546-F557.

15 Mizutani S, Akiyama $\mathrm{H}$, Kurauchi $\mathrm{O}$, et al. In vitro degradation of angiotensin II (A-II) by human placental subcellular fractions, pregnancy sera and purified placental aminopeptidases. Acta Endocrinol 1985;110:135-139.

16 Mizutani S, Okano K, Hasegawa E, et al. Aminopeptidase A in human placenta. Biochem Biophys Acta 1981;678:168-170.

17 Mizutani S, Tomoda Y. Effects of placental proteases on maternal and fetal blood pressure in normal pregnancy and preeclampsia. Am J Hypertens 1996; 9:591-597.

18 Finstad CL, Cordon-Cardo C, Bander NH, et al. Specificity analysis of mouse monoclonal antibodies defining cell surface antigens of human renal cancer. Proc Natl Acad Sci USA 1985;82:2955-2959.

19 Nanus DM, Bogenrieder T, Papandreou CN, et al. Aminopeptidase A expression and enzymatic activity in primary human renal cancers. Int J Oncol 1998; 13:261-267.

20 Ino K, Nagasaka T, Okamoto T, et al. Expression of aminopeptidase A in human gestational choriocarcinoma cell lines and tissues. Placenta 2000;21: 63-72.

21 Fujimura H, Ino K, Nagasaka T, et al. Aminopeptidase A expression in cervical neoplasia and its relationship to neoplastic transformation and progression. Oncology 2000;58:342-352.

22 Shekhar MP, Werdell J, Santner SJ, et al. Breast stroma plays a dominant regulatory role in breast epithelial growth and differentiation: implications for tumor development and progression. Cancer Res 2001;61: 1320-1326.

23 Dong Z, Nemeth JA, Cher ML, et al. Differential regulation of matrix metalloproteinase-9, tissue inhibitor of metalloproteinase-1 (TIMP-1) and TIMP-2 expression in co-cultures of prostate cancer and stromal cells. Int J Cancer 2001;93:507-515.

24 Wang TN, Albo D, Tuszynski GP. Fibroblasts promote breast cancer cell invasion by upregulating tumor matrix metalloproteinase-9 production. Surgery 2002;132:220-225.

25 Boyd RS, Balkwill FR. MMP-2 release and activation in ovarian carcinoma: the role of fibroblasts. Br J Cancer 1999;80:315-321.

26 Ino K, Uehara C, Kikkawa F, et al. Enhancement of aminopeptidase A expression during angiotensin IIinduced choriocarcinoma cell proliferation through AT1 receptor involving protein kinase C- and mitogenactivated protein kinase-dependent signaling pathway. J Clin Endocrinol Metab 2003;88:3973-3982.

27 Kajiyama H, Kikkawa F, Maeda O, et al. Increased expression of dipeptidyl peptidase IV in human mesothelial cells by malignant ascites from ovarian carcinoma patients. Oncology 2002;63:158-165.

28 Ko K, Yazumi S, Yoshikawa K, et al. Activation of fibroblast-derived matrix metalloproteinase-2 by colon-cancer cells in non-contact co-cultures. Int J Cancer 2000;87:165-171.

29 Shibata K, Kikkawa F, Nawa A, et al. Increased matrix metalloproteinase-9 activity in human ovarian cancer cells cultured with conditioned medium from human peritoneal tissue. Clin Exp Metastasis 1997;15: 612-619.

30 Kikkawa F, Nawa A, Shibata K, et al. The different stimulatory effect of normal tissues on the secretion of matrix metalloproteinases and their inhibitors by human ovarian cancer cells. Anticancer Res 1998; 18:4323-4328.

31 Bogenrieder T, Finstad CL, Freeman RH, et al. Expression and localization of aminopeptidase A, aminopeptidase $\mathrm{N}$, and dipeptidyl peptidase IV in benign and malignant human prostate tissue. Prostate 1997;33:225-232.

32 Riemann D, Kehlen A, Thiele $\mathrm{K}$, et al. Induction of aminopeptidase N/CD13 on human lymphocytes after adhesion to fibroblast-like synoviocytes, endothelial 
cells, epithelial cells, and monocytes/macrophages. J Immunol 1997;158:3425-3432.

33 Welch PA, Burrows PD, Namen A, et al. Bone marrow stromal cells and interleukin-7 induce coordinate expression of the BP-1/6C3 antigen and pre-B cell growth. Int Immunol 1990;2:697-705.

34 Wang J, Walker H, Lin Q, et al. The mouse BP-1 gene: structure, chromosomal localization, and regulation of expression by type I interferons and interleukin-7. Genomics 1996;33:167-176.

35 Kehlen A, Gohring B, Langner J, et al. Regulation of the expression of aminopeptidase A, aminopeptidase N/ CD13 and dipeptidyl peptidase IV/CD26 in renal carcinoma cells and renal tubular epithelial cells by cytokines and cAMP-increasing mediators. Clin Exp Immunol 1998;111:435-441.

36 Juillerat-Jeanneret L, Lohm S, Hamou MF, et al. Regulation of aminopeptidase A in human brain tumor vasculature: evidence for a role of transforming growth factor- $\beta$. Lab Invest 2000;80:973-980.

37 Miyashita H, Yamazaki T, Akada T, et al. A mouse orthologue of puromycin-insensitive leucyl-specific aminopeptidase is expressed in endothelial cells and plays an important role in angiogenesis. Blood 2002;99:3241-3249.

38 Touyz RM, Deng LY, He G, et al. Angiotensin II stimulates DNA and protein synthesis in vascular smooth muscle cells from human arteries: role of extracellular signal-regulated kinases. J Hypertens 1999;17:907-916.

39 Nadal JA, Scicli GM, Carbini LA, et al. Angiotensin II stimulates migration of retinal microvascular pericytes: involvement of TGF- $\beta$ and PDGF-BB. Am J Physiol Heart Circ Physiol 2002;282:H739-H748.

40 Fujii H, Nakajima M, Saiki I, et al. Human melanoma invasion and metastasis enhancement by high expression of aminopeptidase N/CD13. Clin Exp Metastasis 1995;13:337-344.

41 Fujimoto J, Sakaguchi H, Hirose R, et al. Expression of vascular endothelial growth factor (VEGF) and its mRNA in uterine cervical cancers. Br J Cancer 1999;80:827-833.

42 Dobbs SP, Hewett PW, Johnson IR, et al. Angiogenesis is associated with vascular endothelial growth factor expression in cervical intraepithelial neoplasia. Br J Cancer 1997;76:1410-1415.

43 Tokumo K, Kodama J, Seki N, et al. Different angiogenic pathways in human cervical cancers. Gynecol Oncol 1998;68:38-44.

44 Cheng WF, Chen CA, Lee CN, et al. Vascular endothelial growth factor in cervical carcinoma. Obstet Gynecol 1999;93:761-765.

45 Guidi AJ, Abu-Jawdeh G, Berse B, et al. Vascular permeability factor (vascular endothelial growth factor) expression and angiogenesis in cervical neoplasia. J Natl Cancer Inst 1995;87:1237-1245. 